\title{
Diagnostic Accuracy of Mean Platelet Volume in the Diagnosis of Acute Coronary Syndromes among Patients with Acute Chest Pain at the Emergency Room of Philippine Heart Center
}

\author{
${ }^{1}$ Zamboanga City Medical Center \\ ${ }^{2}$ Philippine Heart Center
}

Al-Zamzam Abubakar ${ }^{1}$ and Minnie Jane Pineda ${ }^{2}$

\section{ABSTRACT}

Introduction. Mean platelet volume (MPV), an index for platelet size, is believed to be associated with acute coronary syndromes (ACS). This study aims to establish the association of MPV and ACS in the local setting and to further evaluate the diagnostic accuracy of MPV in the detection of ACS.

Methodology. Adult patients presenting with chest pain seen at the ER were submitted for complete blood count (CBC). Specimens were processed for MPV and platelet count using Beckman-Coulter ACT 5Diff hematology auto-analyzer. Patients were grouped into ACS and non-ACS. Independent t-test was used for analysis. Diagnostic cut-off point was determined using Receiver Operating Characteristic (ROC) Curve.

Results. A total of 150 adult patients was examined for MPV and platelet counts. There was a significant difference of MPV between the two groups ( $\mathrm{p}$ value $<0.0001$ ). The MPV of patients with ACS was increased at $8.3 \mathrm{fL}$ compared to $7.3 \mathrm{fL}$ in patients not diagnosed with ACS. At cut-off point of $8.4 \mathrm{fL}$, the positive predictive value and specificity for ACS were $100 \%$, sensitivity of 43.6 and a negative predictive value of 46.2 . The number of platelets was increased in non-ACS group.

Conclusion. The MPV of acute chest patients diagnosed with ACS was significantly higher compared to patients not diagnosed with ACS. Increased MPV at $8.4 \mathrm{fL}$ was highly specific and predictive of ACS. However, the sensitivity and negative predictive value were low. The platelet count of non-ACS group was increased.

Key words : Mean platelet volume, MPV, Acute coronary syndromes, ACS

ISSN 0118-3265

Printed in the Philippines.

Copyright $(9) 2016$ by the PJP.

Received: 23 February 2016.

Accepted: 25 April 2016.

Published online first: 27 April 2016.

http://dx.doi.org/10.21141/PJP.2016.007

Corresponding author: Al-Zamzam A. Abubakar, MD

E-mail: alzamzam_abubakar@yahoo.com

\section{INTRODUCTION}

The clinical use of mean platelet volume (MPV) is unknown. ${ }^{1}$ Although it is routinely measured in hematological auto-analyzers for more than a decade, many laboratories do not usually include this in the final report of complete blood count. The primary reason is the lack of standardization of this value. ${ }^{1,2}$ Another reason is the limited evidence that this measurement adds any valuable information in the clinical situation. ${ }^{1}$

However, there are increasing data attributing MPV with acute coronary syndromes. Mean platelet volume, as an index of platelet size and function, have been found to be increased in patients having disease conditions under the spectrum of acute coronary artery syndromes (ACS). ${ }^{2-7}$ The major reason for this is that increased platelet activity plays a crucial role in the development of acute myocardial infarction. ${ }^{2}$ Though there are well-established risk factors identified in the formation of atherosclerosis that bring about ACS, myocardial infarction (MI) could only and likely to happen if there are large and hyperactive platelets in the circulation. ${ }^{2,3}$

The effective screening of patients at the emergency room for acute coronary syndrome remains a challenge. Currently, cardiac coronary related diseases continue to be the leading cause of morbidity in most of the countries and the number one cause of deaths since the beginning of twentieth century. ${ }^{3}$ The association of increased MPV with a critical disease like ACS may possibly emerge this measurement as a simple and accessible test to estimate platelet 
activity. This will further help to stratify cardiovascular risk among patients with acute coronary syndromes. ${ }^{4}$

The main objective of this study is to determine whether there is an association between mean platelet volume and the diagnosis of acute coronary syndromes in a local setting. We further aim to estimate the diagnostic accuracy of MPV in the detection of acute coronary syndromes in patients with acute chest pain.

\section{METHODOLOGY}

This is a diagnostic accuracy study approved by the Philippine Heart Center- Institutional Ethics Review Board conducted at the emergency room (ER) and ER Point-of-care testing (POCT) satellite laboratory of the Philippine Heart Center with data gathered from May 12, 2012 to July 22, 2012 and from June 1, 2013 to August 31, 2013. Included in the study were consecutive adult ( $\geq 21$ years old) patients with chest pain and informed consent was obtained. Excluded were chest pain patients having serious concurrent illnesses where increase platelet count and mean platelet volume were expected as a result of reactive process from a known injury such as trauma, gastrointestinal bleeding, hyperthyroidism, hematopoietic malignancy, (platelet count $>1,000 \times 109 / \mathrm{L})^{8}$ and sepsis. Patients whose specimens for $\mathrm{CBC}$ are not processed within 15 minutes from blood extraction are likewise not included in the study.

The sample size computed is $\mathrm{n}=150$ at $95 \%$ confidence level with a relative error of $15 \%$ and assumed ACS rate among patients presenting with chest pain at the emergency room of $34.3 \%$ based on the previous study done by Lamorena et al. on ER patients presenting with symptoms suggestive of ACS in the Philippine Heart Center. ${ }^{9}$ The variables of the study included mean platelet volume and platelet count as measured by Beckmann Coulter ACT 5 Diff (Beckmann-Coulter, Inc, Fullerton California) and the diagnoses of these patients. ${ }^{10}$

In the emergency room, the charts of all adult patients who came in due to acute chest pain were reviewed every 8-hour shift for chief complaint and laboratory tests ordered. Informed consent was performed on all eligible patients and was submitted for complete blood count. At least $4 \mathrm{ml}$ of blood was extracted through venipuncture and was collected using a lavender-top (ethelynediaminetriacetic acid/EDTA) vacutainer (BD, Franklin Lakes, NJ USA). The collected specimen in the lavender-top was fed through the manual-loading probe of Beckman Coulter ACT 5Diff hematology auto-analyzer. The result generated by the machine for platelet count and mean platelet volume were recorded. The reference standard followed for all the parameters of complete blood count was based on the Standard Operating Procedure Manual of the Department of Laboratory Medicine (DLM) of Philippine Heart Center. Smears of each of the specimen were performed and stained with Wright's stain to verify and confirm the results generated by the automated analyzer. CBC tests of all qualified patients were performed by licensed medical technologists and trained staff of the Point-of-Care-Testing Section of DLM. Out of 172 patient charts reviewed, 150 patients were included in the study. Informed consent was unable to be performed in twelve (12) patients and ten patients could not be submitted for CBC because they were discharged immediately. The clinical diagnosis of the patients as written by the cardiology fellow trainee on duty at the emergency room was recorded. Admitted patients were followedup for the final diagnosis upon discharge and were the recorded diagnosis for such patients. Diagnoses of patients at the emergency room were based on their medical chart as written by the cardiology fellow trainee who examined them. The basis for the diagnosis of ACS and non-ACS was based solely on what is written by the cardiology fellow trainee at the emergency room. All of the hospital staff involved in the study, including the cardiology fellow trainees and the medical technologists, was blinded during the entire study.

Data was described as mean $\pm \mathrm{SD}$ or frequency and percent distribution comparing the ACS and non-ACS patients. To determine if there is a statistically significant difference of the mean platelet volume and platelet count of these two groups, Independent t-test was used. Cut-off points of MPV were computed with their respective scores of diagnostic accuracy parameters (sensitivity, specificity, positive and negative predictive values), as determined by the area under the curve of Receiver Operating Characteristic (ROC) curve.

\section{RESULTS}

A total of 150 adult patients with chest pain were included in the study and 101 of these patients are diagnosed with acute coronary syndrome. The rest of the chest pain patients in the non-ACS category had heterogeneous diagnoses. These wide variety of diagnoses range from pulmonary problems such as chronic obstructive pulmonary disease, pleural effusion, pneumonia, and pulmonary tuberculosis to other cardiac causes like congestive heart failure, valvular heart diseases and pericardial effusion, and even non-cardiac etiology particularly costochondritis.

Table 1 shows the baseline characteristics of the patients included in the study. Patients with ACS tend to be older. The mean age under ACS category was 62 years old compared to 56 years old in nonACS group. Majority of the population in both groups were males. Hypertension was the most common risk factors seen in 58 patients and significantly associated with the diagnosis of ACS. There were 14 patients who had previous myocardial infarction or stroke and showed a notable relationship with ACS. Aspirin was by far the most common anti-platelet medication used by patients in the study.

The mean platelet volume of patients diagnosed with ACS was remarkably higher than patients who were not diagnosed with acute coronary syndrome at $\mathrm{p}$ value $<0.0001$ (Table 2 ). The ACS patients had an average MPV of 8.3 compared to only 7.3 mean MPV among non-ACS patients. On the other hand, the platelet number of patients not diagnosed with ACS was increased than patients who had a diagnosis of ACS (Table 3). Patients who were not diagnosed with acute coronary syndrome had an average platelet number of 269, 000 in contrast to 235,000 in ACS patients.

The computed cut-off points of mean platelet volume predictive in the diagnosis of acute coronary syndrome are shown in Table 3. The overall diagnostic accuracy of MPV, calculated as the area under the curve by the ROC curve revealed a significant test with a value under the curve of 0.868 and $p$ value less than 0.0001 . At $8.4 \mathrm{fL}$ cut-off result, positive predictive value is $100 \%$ and a $46.2 \%$ negative predictive value. A lower value of $7.3 \mathrm{fL}$ MPV shows a higher negative predictive value of $75 \%$ and $78 \%$ positive predictive value. The cut-off points of MPV between $7.6 \mathrm{fL}$ and $7.8 \mathrm{fL}$ showed fairly acceptable parameters of diagnostic accuracy in detecting acute coronary syndromes. A cut-off value of $7.8 \mathrm{fL}$ satisfactorily predict the diagnosis of ACS having the sensitivity of $80.2 \%$, specificity of $75.5 \%$, positive predictive value of $87.1 \%$ and negative predictive value of $64.9 \%$. Alternatively, a value of $7.7 \mathrm{fL}$ showed relatively similar values of diagnostic accuracy. 


\begin{tabular}{|c|c|c|c|c|c|c|c|}
\hline \multirow{2}{*}{ Characteristics } & \multicolumn{2}{|c|}{$\operatorname{ACS}(n=101)$} & \multicolumn{2}{|c|}{ Non ACS $(n=49)$} & \multicolumn{2}{|r|}{ Total } & \multirow{2}{*}{ p } \\
\hline & $\mathrm{N}$ & $\%$ & $\mathrm{~N}$ & $\%$ & $\mathrm{~N}$ & $\%$ & \\
\hline \multicolumn{8}{|l|}{ Age } \\
\hline Mean + std. dev & & 12.446 & & 18.289 & & $60.67+14.822$ & $0.04 *$ \\
\hline \multicolumn{8}{|l|}{ Sex } \\
\hline Male & 73 & 72.3 & 38 & 77.6 & 111 & 74.0 & \multirow{2}{*}{$0.49 * *$} \\
\hline Female & 28 & 27.7 & 11 & 22.4 & 39 & 26.0 & \\
\hline \multicolumn{8}{|l|}{ Risk factors } \\
\hline Hypertension & 58 & 57.4 & 10 & 20.4 & 68 & 45.3 & $<0.00 * *$ \\
\hline Smoking & 41 & 40.6 & 20 & 40.8 & 61 & 40.7 & $1.00 * *$ \\
\hline Diabetes mellitus & 27 & 26.7 & 8 & 16.3 & 35 & 23.3 & $0.22 * *$ \\
\hline Dyslipidemia & 7 & 6.9 & 2 & 4.1 & 9 & 6.0 & $0.71^{* * *}$ \\
\hline Previous MI / Stroke & 14 & 13.9 & 0 & - & 14 & 9.3 & $0.00 * * *$ \\
\hline \multicolumn{8}{|l|}{ Medication } \\
\hline Aspirin & 24 & 23.8 & 7 & 14.3 & 31 & 20.7 & $0.26 * *$ \\
\hline Clopidogrel & 6 & 5.9 & 3 & 6.1 & 9 & 6.0 & $1.00 * * *$ \\
\hline Warfarin & 0 & - & 1 & 0.2 & 1 & 0.7 & $0.33^{* * *}$ \\
\hline Fondaparinux & 1 & 1.0 & 0 & - & 1 & 0.7 & $1.00 * * *$ \\
\hline
\end{tabular}

*Independent t-test (significant $\mathrm{p}$ value $<0.05) \quad{ }^{* *}$ Chi Square test (significant $\mathrm{p}$ value $<0.05$ ) $\quad{ }^{* * *}$ Fisher's Exact Test (significant $\mathrm{p}$ value $<0.05$ )

Table 2. Mean platelet volume and platelet count according to diagnosis of chest pain (PHC, 2013)

\begin{tabular}{|c|c|c|c|c|c|c|c|}
\hline \multirow{2}{*}{ Characteristics } & \multicolumn{2}{|r|}{ ACS $(n=101)$} & \multicolumn{2}{|r|}{ Non ACS $(n=49)$} & \multicolumn{2}{|r|}{ Total } & \multirow[b]{2}{*}{ p } \\
\hline & $\mathrm{N}$ & $\%$ & $\mathrm{~N}$ & $\%$ & $\mathrm{~N}$ & $\%$ & \\
\hline \multicolumn{8}{|l|}{ MPV (fL) } \\
\hline Mean + std. dev & & $8.279+0.7356$ & & $7.276+0.5356$ & & $7.951+0.8238$ & $<0.00^{*}$ \\
\hline \multicolumn{8}{|l|}{ Platelet Count } \\
\hline Mean + std. dev & & $235.75+74.651$ & & $269.53+98.156$ & & $246.79+84.241$ & $0.02 *$ \\
\hline
\end{tabular}

Table 3. Cut-off points of mean platelet volume predictive of ACS diagnosis

\begin{tabular}{|c|c|c|c|c|c|c|c|}
\hline Platelet Volume (fL) & ACS & Non-ACS & Sn*\% & Sp*\% & PPV* \% & NPV* \% & Kappa Test \\
\hline$>8.4$ & 44 & 0 & 43.6 & 100.0 & 100.0 & 46.2 & $0.335+$ \\
\hline$<8.4$ & 57 & 49 & & & & & 0.052 \\
\hline Total & 101 & 49 & & & & & $<0.0001$ \\
\hline$>7.8$ & 81 & 12 & 80.2 & 75.5 & 87.1 & 64.9 & $0.535+$ \\
\hline$<7.8$ & 20 & 37 & & & & & 0.072 \\
\hline Total & 101 & 49 & & & & & $<0.0001$ \\
\hline$>7.7$ & 83 & 14 & 82.2 & 71.4 & 85.6 & 66.0 & $0.525+$ \\
\hline$<7.7$ & 18 & 35 & & & & & 0.073 \\
\hline Total & 101 & 49 & & & & & $<0.0001$ \\
\hline$>7.6$ & 85 & 18 & 84.2 & 63.3 & 82.5 & 66.0 & $0.479+$ \\
\hline$<7.6$ & 16 & 31 & & & & & 0.077 \\
\hline Total & 101 & 49 & & & & & $<0.0001$ \\
\hline$>7.3$ & 93 & 25 & 92.1 & 49.0 & 78.8 & 75.0 & $0.451+$ \\
\hline$<7.3$ & 8 & 24 & & & & & 0.078 \\
\hline Total & 101 & 49 & & & & & $<0.0001$ \\
\hline
\end{tabular}

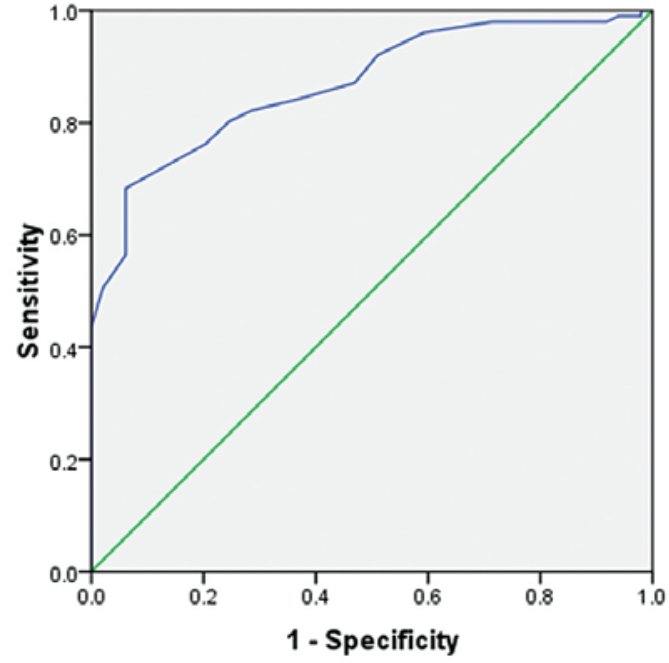

Diagonal segments are produced by ties

Figure 1. The ROC curve of MPV values in association with the diagnosis of ACS (AUC=0.868, Cut-off values 8.4, 7.8, 7.7, 7.6, 7.3).
Figure 1 is the ROC curve showing a significant association between mean platelet volume and the diagnosis of acute coronary syndrome at $\mathrm{p}$ value $<0.0001$. The value under the area is 0.868 .

\section{DISCUSSION}

This study showed that there was an association between MPV and the diagnosis of ACS in patients having chest pain at the ER. The MPV of patients who were diagnosed with acute coronary syndrome was significantly higher compared to patients not diagnosed with ACS. The computed average MPV of patients under ACS group was $8.3 \mathrm{fL}$ while non-ACS patients had $7.3 \mathrm{fL}$. Cut-off points predictive of ACS are calculated based on the area under the curve of the ROC curve revealed a significant test. An MPV of $8.4 \mathrm{fL}$ was highly specific for the diagnosis of ACS.. While the MPV of ACS patients are increased, the number of platelets was significantly decreased compared to non-ACS group. ACS patients are older and composed mostly of males. Hypertension and patients who had previous MI or stroke were also significantly associated with the diagnosis of acute coronary syndromes. 
The size and density of the platelets are markedly heterogeneous in the human circulation. The functional activity of platelets is correlated with their sizes. ${ }^{1,3,7}$ Larger platelets are more likely to be reactive because it contained more granules or active substances that may have important role in coagulation and eventually in thrombus formation and even atherosclerotic plaques. ${ }^{2}$ It is in this proposition that the critical role of the platelets in the pathogenesis of acute coronary syndromes sets in. ${ }^{3}$ This assumption has even made more substantial by the fact that various drugs used in the management of acute coronary syndromes have anti-platelet activities. ${ }^{3}$

With the observation that the activity of the platelets depends on its size and MPV is reliable index of platelet size, many recent studies have hypothesized that there is an increased MPV in patients with acute coronary syndromes. ${ }^{4}$ Though there were several data have already supported this claim, the reason for the increased in the platelet size is not fully understood. ${ }^{2}$ Previous studies have provided several possible explanations. Some authors believed that physiological changes of body metabolism and secretion of biologically active substances as a result of aging or complications of diabetes mellitus and obesity might play a role. ${ }^{2}$ Others are convinced that toxins derived from tobacco smoking and the actual processes during the development of acute coronary events may possibly contribute to the stimulation of bone marrow to produced more larger platelets. ${ }^{2}$ More recent study had shown that platelet size and activity potentially in some ways is genetically determined. ${ }^{2}$ This genomic-wide association study had identified three specific genetic features strongly associated with increased mean platelet volume. ${ }^{2}$ Notice that most of these explanations are all attributed to the known risk factors of acute coronary syndromes such as aging, obesity, diabetes mellitus and cigarette smoking. Consequently, most the available studies had shown positive correlation between increased MPV and these risk factors., ${ }^{2,3,7}$

However, in a large-scale study made by the group of Klovaite, increased MPV is associated with myocardial infarction independent of other known risk factors after an extensive multifactorial adjusted analysis. ${ }^{2}$ This finding is also supported by the study made by Lippi et al. where they concluded that MPV is a useful marker for the risk stratification of ACS patients admitted at the emergency room. ${ }^{4}$

The value of MPV predictive of acute coronary syndromes varies from one study to the other. In this study, the cut off points between $7.6 \mathrm{fL}$ to $7.8 \mathrm{fL}$ had shown satisfactory results of all parameters in diagnostic accuracy of MPV predictive of acute coronary syndromes. This finding is relatively similar to the $7.8 \mathrm{fL}$ cut-off point established by Klovaite et al. with the highest risk of developing myocardial infarction. Higher values are noted in the other studies ranging from $8.0 \mathrm{fL}$ to $10.3 \mathrm{fL} .^{3.7}$ The group of Khandekar have recorded an average MPV of $10.3 \mathrm{fL}$ among MI patients, Lippi and his group showed 8.0 fL cut-off in their result and Mercan et al. have documented values ranging from $8.9 \mathrm{fL}$ to $10.1 \mathrm{fL}$ dependent upon the severity of ischemic conditions in the spectrum of ACS. ${ }^{3,4,7}$ It is also noted that these values are fairly within the range of recommended normal range of MPV in textbooks of laboratory medicine. ${ }^{8}$ Meaning, the increased MPV observed in this study and other previous studies are still within the range of normal plateletsize and not an abnormally large-sized and non-functional platelets usually found in patients with hematolymphoid lesions. ${ }^{8}$

An additional finding in this study is the increased number of platelets of the non-ACS group compared to that of ACS patients.
Theoretically, this result may be explained by consumption of the platelets during the development of acute coronary event. ${ }^{2}$ Thereby, decreasing the actual number of free platelets in the circulation. ${ }^{2}$ In the study made by Klovaite et al., platelet count is not associated with increased risk of myocardial infarction. Either the platelet count is decreased or increased in ACS patients; the number of platelets is not significantly associated with ACS based on previous studies. ${ }^{2}$ The limited number of patients under the non-ACS group may have contributed to this finding. This insufficient number of otherwise control or normal group might be one of the limitations of this study.

Another potential limitation of this study is the sample size. Sample collection was limited by procedures such as CBC not routinely done for chest pain patients at the emergency room and patients complaining of chest pain without significant electrocardiographic findings were quickly discharged. Hence, there were patients who failed to be included in the study. Some of the patients had vague diagnosis because of limited diagnostic work-up brought about by financial constraint. Other probable clinical factor that may affect MPV like the onset of chest pain is not recorded in many of the medical charts of patients included in the study.

Even with a limited population size, the association of increased MPV and diagnosis of acute coronary syndromes is clearly established. From this initial finding, many questions are expected to come out and the existing data presented in this study need to be validated in a larger population. Further investigation in the sizes of the platelets corresponding to the severity of ischemia under the spectrum of disease conditions in ACS would also be beneficial. To explore the correlation of MPV with other known risk factors of ACS such as hypertension, cigarette smoking, diabetes mellitus and others is likewise valuable in the effective risk assessment of chest pain patients at the emergency room. Since platelet activity depends on platelet size as measured through MPV, increased MPV among ACS patients also mean that this test might possibly be an acceptable screening tool in identifying patients who are good candidate for a more expensive platelet function tests like Anti-Xa, P2Y12 and aspirin assays, which are all available in this institution.

\section{CONCLUSION}

The MPV of chest patients diagnosed with acute coronary syndromes was significantly higher compared to patients not diagnosed with ACS. In contrast, the number of platelets in nonACS group was elevated than ACS patients. The calculated average MPV of patients with ACS was $8.3 \mathrm{fL}$ while non-ACS patients had $7.3 \mathrm{fL}$. Cut-off points predictive of ACS were computed based on the area under the curve of the ROC revealed a significant test (AOC is equal to $0.86895 \% \mathrm{CI}(0.812-0.924) \mathrm{p}=<0.0001)$. At cutoff point of $8.4 \mathrm{fL}$, the positive predictive value was highly specific at $100 \%$ and a low negative predictive value at $46 \%$.

\section{AUTHOR DISCLOSURE}

The authors do not have any relevant financial interest in the equipment, products or their respective manufacturers and companies mentioned in this study. Furthermore, there were no funding received from the suppliers or manufactures of all the products included in this study. 


\section{ACKNOWLEDGMENT}

The authors thank the Officer-in-charge of the Division of Laboratory Medicine, Dr. Arlene M. De Luna, for the technical support and invaluable ideas.

The authors also acknowledge the assistance extended by the Chief Medical Technologist of ER POCT Satellite Lab, Mr. Adonis C. Guillon and the Technical Research Board of the Department of Education, Training and Research of Philippine Heart Center.

\section{REFERENCES}

1. Briggs C. Quality counts: new parameters in blood cell counting. Intl J Lab Hematol. 2009;31(3): 277-97. http:// dx.doi.org/10.1111/j.1751-553X.2009.01160.x.

2. Klovaite J, Benn M, Yazdangar S. High platelet volume and increased risk myocardial infarction: 39531 participants from the general population. J Thromb and Haemost. 2011;9(1):4956. http://dx.doi.org/10.1111/j.1538-7836.2010.04110.x.

3. Mercan R, Demir C, Dilek I, Asker M, Atmaca M. Mean platelet volume in acute coronary syndrome. Van Tip Dergisi. 2010;17:89-95.

4. Lippi G, Filippozzi L, Salvagno GL, Montagnana M, Franchini M, Guidi GC, et al. Increased mean platelet volume participants with acute coronary syndromes. Arch Pathol Lab Med. 2009;133(9):1441-3. http://dx.doi.org/10.1043/15432165-133.9.1441. PMID: 19722752.

5. Yilmaz MB, Cihan GC, Guray Y, Guray U, Kisacik HL, et al. Role of mean platelet volume in triagging acute coronary syndromes. J Tromb Thrombolysis. 2008;26(1)49-54. http:// dx.doi.org/10.1007/s11239-007-0078-9.

6. Endler G, Klimesch A, Sunder-Plassmann H, Schillinger M, Exner M, et al. Mean platelet volume in an independent risk factor for myocardial infarction but not for coronary artery disease. Brit J Haematol. 2002;117(2):399-404. http://dx.doi. org/10.1046/j.1365-2141.2002.03441.x.

7. Khandekar MM, Khurana AS, Deshmukh SD, Kakrani AL, Katdare AD. Platelet volume indices in patients with coronary artery disease and acute myocardial infarction: An Indian Scenario. J Clin Path. 2006;59(2):146-9. PMID: 16443728.

8. Vajpayee N, Graham SS, Bem S. Basic examination of blood and bone marrow. In Henry's clinical diagnosis and management by laboratory methods. 22nd ed, ch. 30, pp. 509535.e2. Philadelphia: Elsevier Saunders, 2011.

9. Lamorena N, Torres MCD. Clinical features of emergency department patients presenting with symptoms suggestive of acute coronary syndrome: The Philippine Heart Center Experience 2004. Philippine Heart Center.
10. Coulter ${ }^{\circledR} \operatorname{HmX}$ hematology analyzer with autoloader: operator's guide. CE-Beckmann Coulter, Inc. Fullertone, Ca 92835, 2003.

11. Braunwald E, Antman EM, Beasley JW, et al. ACC/ AHA 2002 guidelines update for the management of patients with unstable angina and non-ST-segment elevation myocardial infarction-2012: summary article. Circulation. 2002;106: 1883-1900. http://dx.doi.org/10.1161/01.CIR. 0000037106.76139 .53 .

\section{APPENDIX}

\section{Definition of Terms}

Acute coronary syndrome (ACS) - a spectrum of clinical conditions ranging from ST-elevation myocardial infarction (MI) to non-ST segment elevation and unstable angina. (Taken from the ACC/ AHA 2002 Guideline Update for the Management of Patients with UA and NSTEMI: A report of the ACC/AHA Task force on Practice Guidelines). ${ }^{11}$

a. ST- elevation MI - presence of a clinical syndrome of acute ischemia with either chest pain or a crescendo pattern of ischemic pain on minimal exertion, plus electrocardiographic ST-segment elevation and/or biomarker evidence of acute ischemic injury (elevated troponin or CK-MB);

b. Non-ST-elevation MI - presence of a clinical syndrome of acute ischemia with either chest rest pain or a crescendo pattern of ischemic pain on minimal exertion, plus electrocardiographic changes and/or biomarker evidence of acute ischemic injury (elevated troponin or CK-MB);

c. Unstable angina - at least one of the following features: (1) angina pectoris occurring at rest (or with min exertion) and usually lasting more than $20 \mathrm{~min}$ (if not interrupted by nitroglycerin), (2) being severe and described as frank pain and of new onset (within 1 month), and (3) occurring with a crescendo pattern (more severe, prolonged, or frequent than previously)

1. Non-Acute Coronary Syndrome (Non-ACS) - refers to clinical conditions of patients having chest pain at the ER other than what are included in the definition of acute coronary syndrome.

2. Mean platelet volume (MPV) - is the arithmetic mean of the extrapolated histogram of the platelets. ${ }^{10}$

3. Platelet count - is the actual count of platelets that are determined using a 64 channel pulse-height analyzer. ${ }^{10}$

Disclaimer: This journal is OPEN ACCESS, providing immediate access to its content on the principle that making research freely available to the public supports a greater global exchange of knowledge. As a requirement for submission to the PJP, all authors have accomplished an AUTHOR FORM, which declares that the ICMJE criteria for authorship have been met by each author listed, that the article represents original material, has not been published, accepted for publication in other journals, or concurrently submitted to other journals, and that all funding and conflicts of interest have been declared. Consent forms have been secured for the publication of information about patients or cases; otherwise, authors have declared that all means have been exhausted for securing consent. 